\title{
Machine Learning for Combinatorial Optimization: a Methodological Tour d'Horizon*
}

\author{
Yoshua Bengio ${ }^{2,3}$, Andrea Lodi ${ }^{1,3}$, and Antoine Prouvost ${ }^{1,3}$ \\ yoshua.bengio@mila.quebec \\ \{andrea.lodi, antoine.prouvost\}@polymtl.ca \\ ${ }^{1}$ Canada Excellence Research Chair in Data Science for Decision \\ Making, École Polytechnique de Montréal \\ ${ }^{2}$ Department of Computer Science and Operations Research, \\ Université de Montréal \\ ${ }^{3}$ Mila, Quebec Artificial Intelligence Institute
}

\begin{abstract}
This paper surveys the recent attempts, both from the machine learning and operations research communities, at leveraging machine learning to solve combinatorial optimization problems. Given the hard nature of these problems, state-of-the-art algorithms rely on handcrafted heuristics for making decisions that are otherwise too expensive to compute or mathematically not well defined. Thus, machine learning looks like a natural candidate to make such decisions in a more principled and optimized way. We advocate for pushing further the integration of machine learning and combinatorial optimization and detail a methodology to do so. A main point of the paper is seeing generic optimization problems as data points and inquiring what is the relevant distribution of problems to use for learning on a given task.
\end{abstract}

\section{Introduction}

Operations research, also referred to as prescriptive analytics, started in the second world war as an initiative to use mathematics and computer science

${ }^{*}$ Accepted to the European Journal of Operations Research. (C)2020. Licensed under the Creative Commons (c) (1) $€$ 
to assist military planners in their decisions (Fortun and Schweber, 1993). Nowadays, it is widely used in the industry, including but not limited to transportation, supply chain, energy, finance, and scheduling. In this paper, we focus on discrete optimization problems formulated as integer constrained optimization, i.e., with integral or binary variables (called decision variables). While not all such problems are hard to solve (e.g., shortest path problems), we concentrate on combinatorial optimization (CO) problems (NP-hard). This is bad news, in the sense that, for those problems, it is considered unlikely that an algorithm whose running time is polynomial in the size of the input exists. However, in practice, $\mathrm{CO}$ algorithms can solve instances with up to millions of decision variables and constraints.

How is it possible to solve NP-hard problems in practical time? Let us look at the example of the traveling salesman problem (TSP), a NP-hard problem defined on a graph where we are searching for a cycle of minimum length visiting once and only once every node. A particular case is that of the Euclidian TSP. In this version, each node is assigned coordinates in a plane 1 and the cost on an edge connecting two nodes is the Euclidian distance between them. While theoretically as hard as the general TSP, good approximate solution can be found more efficiently in the Euclidian case by leveraging the structure of the graph (Larson and Odoni, 1981, Chapter 6.4.7). Likewise, diverse types of problems are solved by leveraging their special structure. Other algorithms, designed to be general, are found in hindsight to be empirically more efficient on particular problems types. The scientific literature covers the rich set of techniques researchers have developed to tackle different $\mathrm{CO}$ problems. An expert will know how to further refine algorithm parameters to different behaviors of the optimization process, thus extending this knowledge with unwritten intuition. These techniques, and the parameters controlling them, have been collectively learned by the community to perform on the inaccessible distribution of problem instances deemed valuable. The focus of this paper is on $\mathrm{CO}$ algorithms that automatically perform learning on a chosen implicit distribution of problems. Incorporating machine learning (ML) components in the algorithm can achieve this.

Conversely, ML focuses on performing a task given some (finite and usually noisy) data. It is well suited for natural signals for which no clear mathematical formulation emerges because the true data distribution is not known analytically, such as when processing images, text, voice or molecules, or with recommender systems, social networks or financial predictions. Most

\footnotetext{
${ }^{1}$ Or more generally in a vector space of arbitrary dimension.
} 
of the times, the learning problem has a statistical formulation that is solved through mathematical optimization. Recently, dramatic progress has been achieved with deep learning, an ML sub-field building large parametric approximators by composing simpler functions. Deep learning excels when applied in high dimensional spaces with a large number of data points.

\subsection{Motivation}

From the CO point of view, machine learning can help improve an algorithm on a distribution of problem instances in two ways. On the one side, the researcher assumes expert knowledg 2 about the optimization algorithm, but wants to replace some heavy computations by a fast approximation. Learning can be used to build such approximations in a generic way, i.e., without the need to derive new explicit algorithms. On the other side, expert knowledge may not be sufficient and some algorithmic decisions may be unsatisfactory. The goal is therefore to explore the space of these decisions, and learn out of this experience the best performing behavior (policy), hopefully improving on the state of the art. Even though ML is approximate, we will demonstrate through the examples surveyed in this paper that this does not systematically mean that incorporating learning will compromise overall theoretical guarantees. From the point of view of using ML to tackle a combinatorial problem, $\mathrm{CO}$ can decompose the problem into smaller, hopefully simpler, learning tasks. The $\mathrm{CO}$ structure therefore acts as a relevant prior for the model. It is also an opportunity to leverage the CO literature, notably in terms of theoretical guarantees (e.g., feasibility and optimality).

\section{$1.2 \quad$ Setting}

Imagine a delivery company in Montreal that needs to solve TSPs. Every day, the customers may vary, but usually, many are downtown and few on top of the Mont Royal mountain. Furthermore, Montreal streets are laid on a grid, making the distances close to the $\ell_{1}$ distance. How close? Not as much as Phoenix, but certainly more than Paris. The company does not care about solving all possible TSPs, but only theirs. Explicitly defining what makes a TSP a likely one for the company is tedious, does not scale, and it is not clear how it can be leveraged when explicitly writing an optimization algorithm. We would like to automatically specialize TSP algorithms for this company.

\footnotetext{
${ }^{2}$ Theoretical and/or empirical.
} 
The true probability distribution of likely TSPs in the Montreal scenario is defining the instances on which we would like our algorithm to perform well. This is unknown, and cannot even be mathematically characterized in an explicit way. Because we do not know what is in this distribution, we can only learn an algorithm that performs well on a finite set of TSPs sampled from this distribution (for instance, a set of historical instances collected by the company), thus implicitly incorporating the desired information about the distribution of instances. As a comparison, in traditional ML tasks, the true distribution could be that of all possible images of cats, while the training distribution is a finite set of such images. The challenge in learning is that an algorithm that performs well on problem instances used for learning may not work properly on other instances from the true probability distribution. For the company, this would mean the algorithm only does well on past problems, but not on the future ones. To control this, we monitor the performance of the learned algorithm over another independent set of unseen problem instances. Keeping the performances similar between the instances used for learning and the unseen ones is known in ML as generalizing. Current ML algorithms can generalize to examples from the same distribution, but tend to have more difficulty generalizing out-ofdistribution (although this is a topic of intense research in ML), and so we may expect $\mathrm{CO}$ algorithms that leverage ML models to fail when evaluated on unseen problem instances that are too far from what has been used for training the ML predictor. As previously motivated, it is also worth noting that traditional $\mathrm{CO}$ algorithms might not even work consistently across all possible instances of a problem family, but rather tend to be more adapted to particular structures of problems, e.g., Euclidean TSPs.

Finally, the implicit knowledge extracted by ML algorithms is complementary to the hard-won explicit expertise extracted through CO research. Rather, it aims to augment and automate the unwritten expert intuition (or lack of) on various existing algorithms. Given that these problems are highly structured, we believe it is relevant to augment solving algorithms with machine learning - and especially deep learning to address the high dimensionality of such problems.

In the following, we survey the attempts in the literature to achieve such automation and augmentation, and we present a methodological overview of those approaches. In light of the current state of the field, the literature we survey is exploratory, i.e., we aim at highlighting promising research directions in the use of $\mathrm{ML}$ within $\mathrm{CO}$, instead of reporting on already mature algorithms. 


\subsection{Outline}

We have introduced the context and motivations for building combinatorial optimization algorithms together with machine learning. The remainder of this paper is organized as follows. Section 2 provides minimal prerequisites in combinatorial optimization, machine learning, deep learning, and reinforcement learning necessary to fully grasp the content of the paper. Section 3 surveys the recent literature and derives two distinctive, orthogonal, views: Section 3.1 shows how machine learning policies can either be learned by imitating an expert or discovered through experience, while Section 3.2 discusses the interplay between the ML and CO components. Section 5 pushes further the reflection on the use of ML for combinatorial optimization and brings to the fore some methodological points. In Section 6, we detail critical practical challenges of the field. Finally, some conclusions are drawn in Section 7.

\section{Preliminaries}

In this section, we give a basic (sometimes rough) overview of combinatorial optimization and machine learning, with the unique aim of introducing concepts that are strictly required to understand the remainder of the paper.

\subsection{Combinatorial Optimization}

Without loss of generality, a CO problem can be formulated as a constrained min-optimization program. Constraints model natural or imposed restrictions of the problem, variables define the decisions to be made, while the objective function, generally a cost to be minimized, defines the measure of the quality of every feasible assignment of values to variables. If the objective and constraints are linear, the problem is called a linear programming (LP) problem. If, in addition, some variables are also restricted to only assume integer values, then the problem is a mixed-integer linear programming (MILP) problem.

The set of points that satisfy the constraints is the feasible region. Every point in that set (often referred to as a feasible solution) yields an upper bound on the objective value of the optimal solution. Exact solving is an important aspect of the field, hence a lot of attention is also given to find lower bounds to the optimal cost. The tighter the lower bounds, with respect to the optimal solution value, the higher the chances that the current algorithmic approaches to tackle mixed-integer linear programmings (MILPs) 
described in the following could be successful, i.e., effective if not efficient.

Linear and mixed-integer linear programming problems are the workhorse of $\mathrm{CO}$ because they can model a wide variety of problems and are the best understood, i.e., there are reliable algorithms and software tools to solve them. We give them special considerations in this paper but, of course, they do not represent the entire $\mathrm{CO}$, mixed-integer nonlinear programming being a rapidly expanding and very significant area both in theory and in practical applications. With respect to complexity and solution methods, LP is a polynomial problem, well solved, in theory and in practice, through the simplex algorithm or interior points methods. Mixed-integer linear programming, on the other hand, is an NP-hard problem, which does not make it hopeless. Indeed, it is easy to see that the complexity of MILP is associated with the integrality requirement on (some of) the variables, which makes the MILP feasible region nonconvex. However, dropping the integrality requirement (i) defines a proper relaxation of MILP (i.e., an optimization problem whose feasible region contains the MILP feasible region), which (ii) happens to be an LP, i.e., polynomially solvable. This immediately suggests the algorithmic line of attack that is used to solve MILP through a whole ecosystem of branch-and-bound (B\&B) techniques to perform implicit enumeration. Branch and bound implemements a divide-and-conquer type of algorithm representable by a search tree in which, at every node, an LP relaxation of the problem (possibly augmented by branching decisions, see below) is efficiently computed. If the relaxation is infeasible, or if the solution of the relaxation is naturally (mixed-)integer, i.e., MILP feasible, the node does not need to be expanded. Otherwise, there exists at least one variable, among those supposed to be integer, taking a fractional value in the LP solution and that variable can be chosen for branching (enumeration), i.e., by restricting its value in such a way that two child nodes are created. The two child nodes have disjoint feasible regions, none of which contains the solution of the previous LP relaxation. We use Figure 1 to illustrate the $\mathrm{B} \& \mathrm{~B}$ algorithm for a minimization MILP. At the root node in the figure, the variable $x_{2}$ has a fractional value in the LP solution (not represented), thus branching is done on the floor (here zero) and ceiling (here one) of this value. When an integer solution is found, we also get an upper bound (denoted as $\bar{z}$ ) on the optimal solution value of the problem. At every node, we can then compare the solution value of the relaxation (denoted as $\underline{z}$ ) with the minimum upper bound found so far, called the incumbent solution value. If the latter is smaller than the former for a specific node, no better (mixed-)integer solution can be found in the sub-tree originated by the node itself, and it can be pruned. 


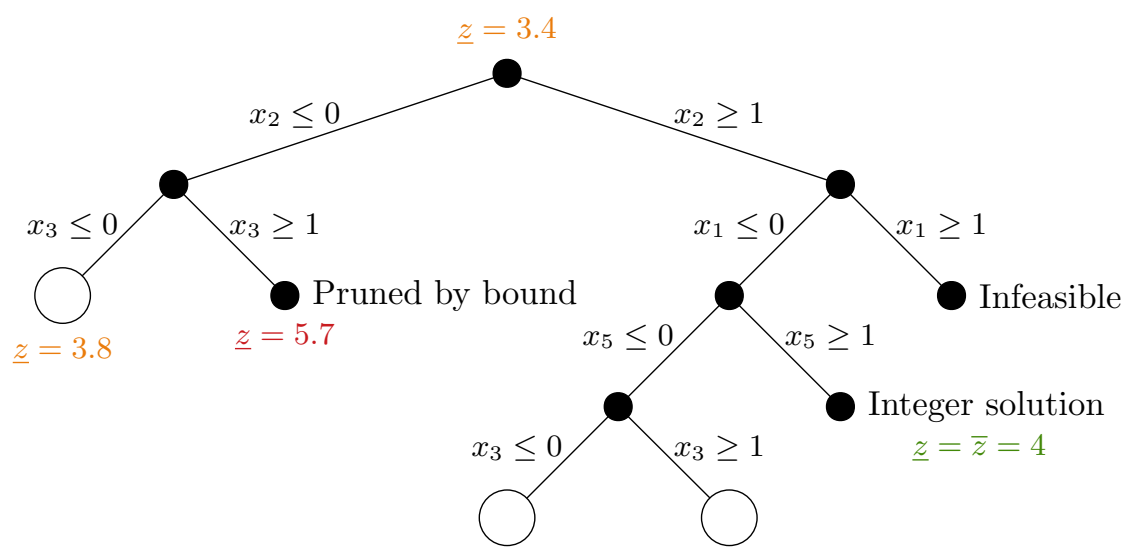

Figure 1: A branch-and-bound tree for MILPs. The LP relaxation is computed at every node (only partially shown in the figure). Nodes still open for exploration are represented as blank.

All commercial and noncommercial MILP solvers enhance the above enumeration framework with the extensive use of cutting planes, i.e., valid linear inequalities that are added to the original formulation (especially at the root of the $\mathrm{B} \& \mathrm{~B}$ tree) in the attempt of strengthening its LP relaxation. The resulting framework, referred to as the branch-and-cut algorithm, is then further enhanced by additional algorithmic components, preprocessing and primal heuristics being the most crucial ones. The reader is referred to Wolsey (1998) and Conforti et al. (2014) for extensive textbooks on MILP and to Lodi (2009) for a detailed description of the algorithmic components of the MILP solvers.

We end the section by noting that there is a vast literature devoted to (primal) heuristics, i.e., algorithms designed to compute "good in practice" solutions to CO problems without optimality guarantee. Although a general discussion on them is outside the scope here, those heuristic methods play a central role in $\mathrm{CO}$ and will be considered in specific contexts in the present paper. The interested reader is referred to Fischetti and Lodi (2011) and Gendreau and Potvin (2010).

\subsection{Machine Learning}

Supervised learning In supervised learning, a set of input (features) / target pairs is provided and the task is to find a function that for every input has a predicted output as close as possible to the provided target. Finding such a function is called learning and is solved through an optimization 
problem over a family of functions. The loss function, i.e., the measure of discrepancy between the output and the target, can be chosen depending on the task (regression, classification, etc.) and on the optimization methods. However, this approach is not enough because the problem has a statistical nature. It is usually easy enough to achieve a good score on the given examples but one wants to achieve a good score on unseen examples (test data). This is known as generalization.

Mathematically speaking, let $X$ and $Y$, following a joint probability distribution $P$, be random variables representing the input features and the target. Let $\ell$ be the per sample loss function to minimize, and let $\left\{f_{\theta} \mid \theta \in\right.$ $\left.\mathbb{R}^{p}\right\}$ be the family of ML models (parametric in this case) to optimize over. The supervised learning problem is framed as

$$
\min _{\theta \in \mathbb{R}^{p}} \mathbb{E}_{X, Y \sim P} \ell\left(Y, f_{\theta}(X)\right) .
$$

For instance, $f_{\theta}$ could be a linear model with weights $\theta$ that we wish to learn. The loss function $\ell$ is task dependent (e.g., classification error) and can sometimes be replaced by a surrogate one (e.g., a differentiable one). The probability distribution is unknown and inaccessible. For example, it can be the probability distribution of all natural images. Therefore, it is approximated by the empirical probability distribution over a finite dataset $D_{\text {train }}=\left\{\left(x_{i}, y_{i}\right)\right\}_{i}$ and the optimization problem solved is

$$
\min _{\theta \in \mathbb{R}^{p}} \sum_{(x, y) \in D_{\text {train }}} \frac{1}{\left|D_{\text {train }}\right|} \ell\left(y, f_{\theta}(x)\right) .
$$

A model is said to generalize, if low objective values of (2) translate in low objective values of (11). Because (11) remains inaccessible, we estimate the generalization error by evaluating the trained model on a separate test dataset $D_{\text {test }}$ with

$$
\sum_{(x, y) \in D_{\text {test }}} \frac{1}{\left|D_{\text {test }}\right|} \ell\left(y, f_{\theta}(x)\right) .
$$

If a model (i.e., a family of functions) can represent many different functions, the model is said to have high capacity and is prone to overfitting: doing well on the training data but not generalizing to the test data. Regularization is anything that can improve the test score at the expense of the training score and is used to restrict the practical capacity of a model. On the contrary, if the capacity is too low, the model underfits and performs poorly on both sets. The boundary between overfitting and underfitting can be estimated by changing the effective capacity (the richness of the family of functions 
reachable by training): below the critical capacity one underfits and test error decreases with increases in capacity, while above that critical capacity one overfits and test error increases with increases in capacity.

Selecting the best among various trained models cannot be done on the test set. Selection is a form of optimization, and doing so on the test set would bias the estimator in (2). This is a common form of data dredging, and a mistake to be avoided. To perform model selection, a validation dataset $D_{\text {valid }}$ is used to estimate the generalization error of different ML models is necessary. Model selection can be done based on these estimates, and the final unbiased generalization error of the selected model can be computed on the test set. The validation set is therefore often used to select effective capacity, e.g., by changing the amount of training, the number of parameters $\theta$, and the amount of regularization imposed to the model.

Unsupervised learning In unsupervised learning, one does not have targets for the task one wants to solve, but rather tries to capture some characteristics of the joint distribution of the observed random variables. The variety of tasks include density estimation, dimensionality reduction, and clustering. Because unsupervised learning has received so far little attention in conjunction with $\mathrm{CO}$ and its immediate use seems difficult, we are not discussing it any further. The reader is referred to Bishop (2006); Murphy (2012); Goodfellow et al. (2016) for textbooks on machine learning.

Reinforcement learning In reinforcement learning (RL), an agent interacts with an environment through a markov decision process (MDP), as illustrated in Figure 2. At every time step, the agent is in a given state of the environment and chooses an action according to its (possibly stochastic) policy. As a result, it receives from the environment a reward and enters a new state. The goal in RL is to train the agent to maximize the expected sum of future rewards, called the return. For a given policy, the expected return given a current state (resp. state and action pair) is known as the value function (resp. state action value function). Value functions follow the Bellman equation, hence the problem can be formulated as dynamic programming, and solved approximately. The dynamics of the environment need not be known by the agent and are learned directly or indirectly, yielding an exploration $v s$ exploitation dilemma: choosing between exploring new states for refining the knowledge of the environment for possible long-term improvements, or exploiting the best-known scenario learned so far (which tends to be in already visited or predictable states). 


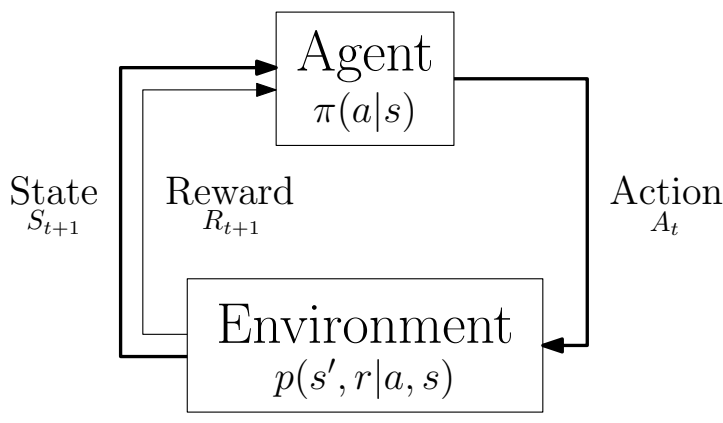

Figure 2: The Markov decision process associated with reinforcement learning, modified from Sutton and Barto (2018). The agent behavior is defined by its policy $\pi$, while the environment evolution is defined by the dynamics $p$. Note that the reward is not necessary to define the evolution and is provided only as a learning mechanism for the agent. Actions, states, and rewards are random variables in the general framework.

The state should fully characterize the environment at every step, in the sense that future states only depend on past states via the current state (the Markov property). When this is not the case, similar methods can be applied but we say that the agent receives an observation of the state. The Markov property no longer holds and the MDP is said to be partially observable.

Defining a reward function is not always easy. Sometimes one would like to define a very sparse reward, such as 1 when the agent solves the problem, and 0 otherwise. Because of its underlying dynamic programming process, RL is naturally able to credit states/actions that lead to future rewards. Nonetheless, the aforementioned setting is challenging as it provides no learning opportunity until the agent (randomly, or through advanced approaches) solves the problem. Furthermore, when the policy is approximated (for instance, by a linear function), the learning is not guaranteed to converge and may fall into local minima. For example, an autonomous car may decide not to drive anywhere for fear of hitting a pedestrian and receiving a dramatic negative reward. These challenges are strongly related to the aforementioned exploration dilemma. The reader is referred to Sutton and Barto (2018) for an extensive textbook on reinforcement learning.

Deep learning Deep learning is a successful method for building parametric composable functions in high dimensional spaces. In the case of the simplest neural network architecture, the feedforward neural network 
(also called an multilayer perceptron (MLP)), the input data is successively passed through a number of layers. For every layer, an affine transformation is applied on the input vector, followed by a non-linear scalar function (named activation function) applied element-wise. The output of a layer, called intermediate activations, is passed on to the next layer. All affine transformations are independent and represented in practice as different matrices of coefficients. They are learned, i.e., optimized over, through stochastic gradient descent (SGD), the optimization algorithm used to minimize the selected loss function. The stochasticity comes from the limited number of data points used to compute the loss before applying a gradient update. In practice, gradients are computed using reverse mode automatic differentiation, a practical algorithm based on the chain rule, also known as back-propagation. Deep neural networks can be difficult to optimize, and a large variety of techniques have been developed to make the optimization behave better, often by changing architectural designs of the network. Because neural networks have dramatic capacities, i.e., they can essentially match any dataset, thus being prone to overfitting, they are also heavily regularized. Training them by SGD also regularizes them because of the noise in the gradient, making neural networks generally robust to overfitting issues, even when they are very large and would overfit if trained with more aggressive optimization. In addition, many hyper-parameters exist and different combinations are evaluated (known as hyper-parameters optimization). Deep learning also sets itself apart from more traditional ML techniques by taking as inputs all available raw features of the data, e.g., all pixels of an image, while traditional ML typically requires to engineer a limited number of domain-specific features.

Deep learning researchers have developed different techniques to tackle this variety of structured data in a manner that can handle variable-size data structures, e.g., variable-length sequences. In this paragraph, and in the next, we present such state-of-the-art techniques. These are complex topics, but lack of comprehension does not hinder the reading of the paper. At a high level, it is enough to comprehend that these are architectures designed to handle different structures of data. Their usage, and in particular the way they are learned, remains very similar to plain feedforward neural networks introduced above. The first architectures presented are the recurrent neural networks (RNNs). These models can operate on sequence data by sharing parameters across different sequence steps. More precisely, a same neural network block is successively applied at every step of the sequence, i.e., with the same architecture and parameter values at each time step. The specificity of such a network is the presence of recurrent layers: 
layers that take as input both the activation vector of the previous layer and its own activation vector on the preceding sequence step (called a hidden state vector), as illustrated in Figure 3 .
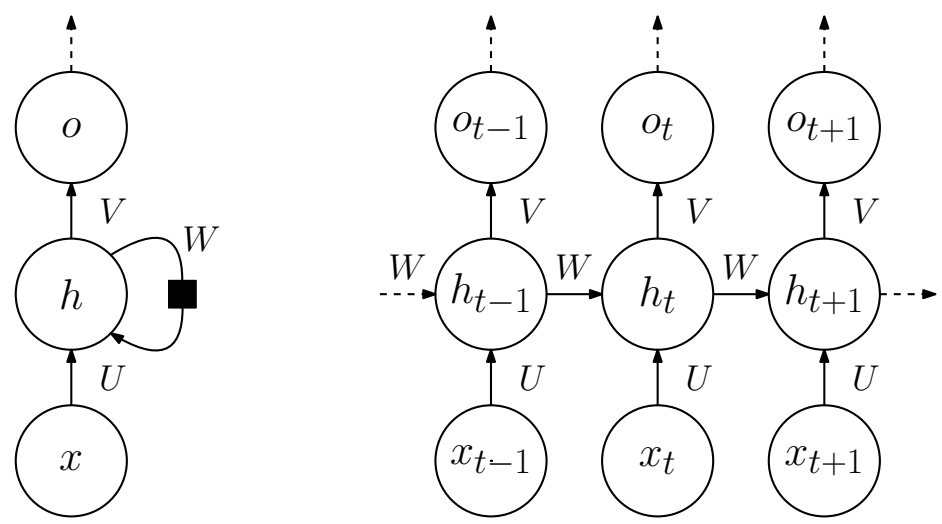

Figure 3: A vanilla RNN modified from Goodfellow et al. (2016). On the left, the black square indicates a one step delay. On the right, the same RNN is shown unfolded. Three sets $U, V$, and $W$ of parameters are represented and re-used at every time step.

Another important size-invariant technique are attention mechanisms. They can be used to process data where each data point corresponds to a set. In that context, parameter sharing is used to address the fact that different sets need not to be of the same size. Attention is used to query information about all elements in the set, and merge it for downstream processing in the neural network, as depicted in Figure 4. An affinity function takes as input the query (which represents any kind of contextual information which informs where attention should be concentrated) and a representation of an element of the set (both are activation vectors) and outputs a scalar. This is repeated over all elements in the set for the same query. Those scalars are normalized (for instance with a softmax function) and used to define a weighted sum of the representations of elements in the set that can, in turn, be used in the neural network making the query. This form of content-based soft attention was introduced by Bahdanau et al. (2015). A general explanation of attention mechanisms is given by Vaswani et al. (2017). Attention can be used to build graph neural networks (GNNs), i.e., neural networks able to process graph structured input data, as done by Veličković et al. (2018). In this architecture, every node attends over the set of its neighbors. The process is repeated multiple times to gather 
information about nodes further away. GNNs can also be understood as a form of message passing (Gilmer et al., 2017).

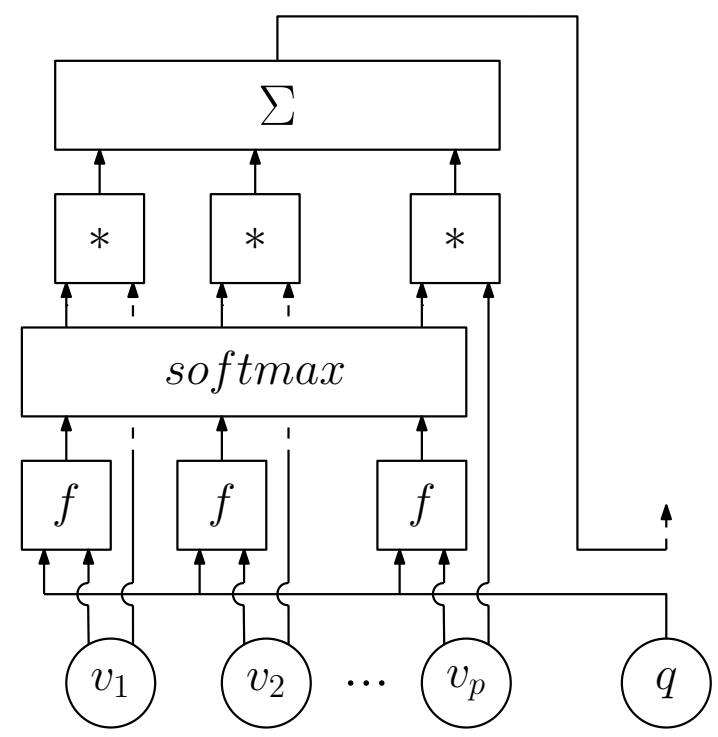

Figure 4: A vanilla attention mechanism where a query $q$ is computed against a set of values $\left(v_{i}\right)_{i}$. An affinity function $f$, such as a dot product, is used on query and value pairs. If it includes some parameters, the mechanism can be learned.

Deep learning and back-propagation can be used in supervised, unsupervised, or reinforcement learning. The reader is referred to Goodfellow et al. (2016) for a machine learning textbook devoted to deep learning.

\section{$3 \quad$ Recent approaches}

We survey different uses of ML to help solve combinatorial optimization problems and organize them along two orthogonal axes. First, in Section 3.1 we illustrate the two main motivations for using learning: approximation and discovery of new policies. Then, in Section 3.2, we show examples of different ways to combine learned and traditional algorithmic elements.

\subsection{Learning methods}

This section relates to the two motivations reported in Section 1.1 for using $\mathrm{ML}$ in CO. In some works, the researcher assumes theoretical and/or 
empirical knowledge about the decisions to be made for a $\mathrm{CO}$ algorithm, but wants to alleviate the computational burden by approximating some of those decisions with machine learning. On the contrary, we are also motivated by the fact that, sometimes, expert knowledge is not satisfactory and the researcher wishes to find better ways of making decisions. Thus, ML can come into play to train a model through trial and error reinforcement learning.

We frame both these motivations in the state/action MDP framework introduced in section 2.2, where the environment is the internal state of the algorithm. We care about learning algorithmic decisions utilized by a $\mathrm{CO}$ algorithm and we call the function making the decision a policy, that, given all available information 3 returns (possibly stochastically) the action to be taken. The policy is the function that we want to learn using ML and we show in the following how the two motivations naturally yield two learning settings. Note that the case where the length of the trajectory of the MDP has value 1 is a common edge case (called the bandit setting) where this formulation can seem excessive, but it nonetheless helps comparing methods.

In the case of using ML to approximate decisions, the policy is often learned by imitation learning, thanks to demonstrations, because the expected behavior is shown (demonstrated) to the ML model by an expert (also called oracle, even though it is not necessarily optimal), as shown in Figure 5. In this setting, the learner is not trained to optimize a performance measure, but to blindly mimic the expert.

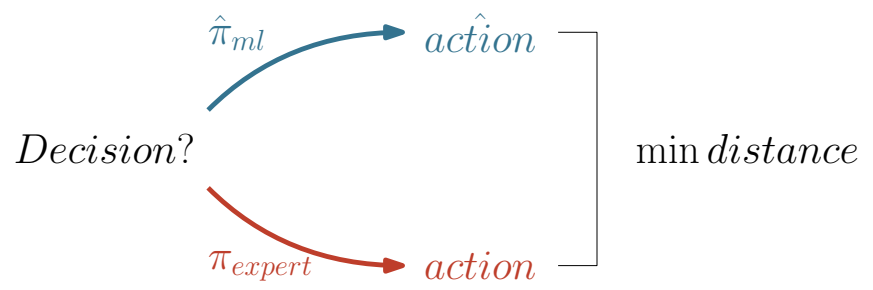

Figure 5: In the demonstration setting, the policy is trained to reproduce the action of an expert policy by minimizing some discrepancy in the action space.

In the case where one cares about discovering new policies, i.e., optimizing an algorithmic decision function from the ground up, the policy may be learned by reinforcement learning through experience, as shown in Fig-

\footnotetext{
${ }^{3}$ A state if the information is sufficient to fully characterize the environment at that time in a Markov decision process setting.
} 
ure 6. Even though we present the learning problem under the fundamental MDP of RL, this does not constrain one to use the major RL algorithms (approximate dynamic programming and policy gradients) to maximize the expected sum of rewards. Alternative optimization methods, such as bandit algorithms, genetic algorithms, direct/local search, can also be used to solve the RL problem 4

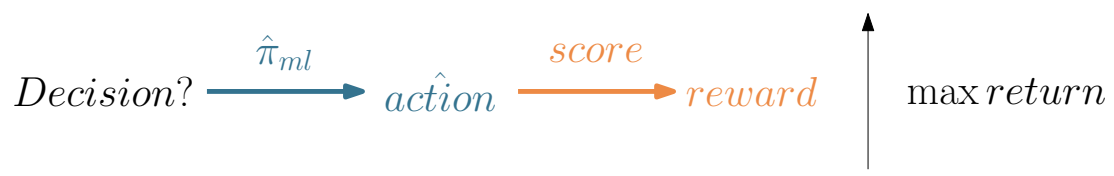

Figure 6: When learning through a reward signal, no expert is involved; only maximizing the expected sum of future rewards (the return) matters.

It is critical to understand that in the imitation setting, the policy is learned through supervised targets provided by an expert for every action (and without a reward), whereas in the experience setting, the policy is learned from a reward (possibly delayed) signal using RL (and without an expert). In imitation, the agent is taught what to do, whereas in RL, the agent is encouraged to quickly accumulate rewards. The distinction between these two settings is far more complex than the distinction made here. We explore some of this complexity, including their strengths and weaknesses, in Section 5.1.

In the following, few papers demonstrating the different settings are surveyed.

\subsubsection{Demonstration}

In Baltean-Lugojan et al. (2018), the authors use a neural network to approximate the lower bound improvement generated by tightening the current relaxation via cutting planes (cuts, for short). More precisely, Baltean-Lugojan et al. (2018) consider non-convex quadratic programming problems and aim at approximating the associated semidefinite programming (SDP) relaxation, known to be strong but time-consuming, by a linear program. A straightforward way of doing that is to iteratively add (linear) cutting planes associated with negative eigenvalues, especially considering small-size (square) submatrices of the original quadratic objective function. That approach has the

\footnotetext{
${ }^{4}$ In general, identifying which algorithm will perform best is an open research question unlikely to have a simple answer, and is outside of the scope of the methodology presented here.
} 
advantage of generating sparse cut:5 but it is computationally challenging because of the exponential number of those submatrices and because of the difficulty of finding the right metrics to select among the violated cuts. The authors propose to solve SDPs to compute the bound improvement associated with considering specific submatrices, which is also a proxy on the quality of the cuts that could be separated from the same submatrices. In this context, supervised (imitation) learning is applied offline to approximate the objective value of the SDP problem associated with a submatrix selection and, afterward, the model can be rapidly applied to select the most promising submatrices without the very significant computational burden of solving SDPs. Of course, the rational is that the most promising submatrices correspond to the most promising cutting planes and Baltean-Lugojan et al. (2018) train a model to estimate the objective of an SDP problem only in order to decide to add the most promising cutting planes. Hence, cutting plane selection is the ultimate policy learned.

Another example of demonstration is found in the context of branching policies in $\mathrm{B} \& \mathrm{~B}$ trees of MILPs. The choice of variables to branch on can dramatically change the size of the $\mathrm{B} \& \mathrm{~B}$ tree, hence the solving time. Among many heuristics, a well-performing approach is strong branching (Applegate et al., 2007). Namely, for every branching decision to be made, strong branching performs a one step look-ahead by tentatively branching on many candidate variables, computes the LP relaxations to get the potential lower bound improvements, and eventually branches on the variable providing the best improvement. Even if not all variables are explored, and the LP value can be approximated, this is still a computationally expensive strategy. For these reasons, Marcos Alvarez et al. (2014, 2017) use a special type of decision tree (a classical model in supervised learning) to approximate strong branching decisions using supervised learning. Khalil et al. (2016) propose a similar approach, where a linear model is learned on the fly for every instance by using strong branching at the top of the tree, and eventually replacing it by its ML approximation. The linear approximator of strong branching introduced in Marcos Alvarez et al. (2016) is learned in an active fashion: when the estimator is deemed unreliable, the algorithm falls back to true strong branching and the results are then used for both branching and learning. In all the branching algorithms presented here, inputs to the ML model are engineered as a vector of fixed length with static features descriptive of the instance, and dynamic features providing

\footnotetext{
${ }^{5}$ The reader is referred to Dey and Molinaro (2018) for a detailed discussion on the importance of sparse cutting planes in MILP.
} 
information about the state of the B\&B process. Gasse et al. (2019) use a neural network to learn an offline approximation to strong branching, but, contrary to the aforementioned papers, the authors use a raw exhaustive representation (i.e., they do not discard nor aggregate any information) of the sub-problem associated with the current branching node as input to the ML model. Namely, an MILP sub-problem is represented as a bipartite graph on variables and constraints, with edges representing non-zero coefficients in the constraint matrix. Each node is augmented with a set of features to fully describe the sub-problem, and a GNN is used to build an ML approximator able to process this type of structured data. Node selection, i.e., deciding on the next branching node to explore in a $\mathrm{B} \& \mathrm{~B}$ tree, is also a critical decision in MILP. He et al. (2014) learn a policy to select among the open branching nodes the one that contains the optimal solution in its sub-tree. The training algorithm is an online learning method collecting expert behaviors throughout the entire learning phase. The reader is referred to Lodi and Zarpellon (2017) for an extended survey on learning and branching in MILPs.

Branch and bound is a technique not limited to MILP and can be use for general tree search. Hottung et al. (2017) build a tree search procedure for the container pre-marshalling problem in which they aim to learn, not only a branching policy (similar in principle to what has been discussed in the previous paragraph), but also a value network to estimate the value of partial solutions and used for bounding. The authors leverage a form of convolutional neural network $(\mathrm{CNN}) 6$ for both networks and train them in a supervised fashion using pre-computed solutions of the problem. The resulting algorithm is heuristic due the approximations made while bounding.

As already mentioned at the beginning of Section 3.1, learning a policy by demonstration is identical to supervised learning, where training pairs of input state and target actions are provided by the expert. In the simplest case, expert decisions are collected beforehand, but more advanced methods can collect them online to increase stability as previously shown in Marcos Alvarez et al. (2016) and He et al. (2014).

\subsubsection{Experience}

Considering the TSP on a graph, it is easy to devise a greedy heuristic that builds a tour by sequentially picking the nodes among those that have not been visited yet, hence defining a permutation. If the criterion for selecting

\footnotetext{
${ }^{6}$ A type of neural network, usually used on image input, that leverages parameter sharing to extract local information.
} 
the next node is to take the closest one, then the heuristic is known as the nearest neighbor. This simple heuristic has poor practical performance and many other heuristics perform better empirically, i.e., build cheaper tours. Selecting the nearest node is a fair intuition but turns out to be far from satisfactory. Khalil et al. (2017a) suggest learning the criterion for this selection. They build a greedy heuristic framework, where the node selection policy is learned using a GNN (Dai et al., 2016), a type of neural network able to process input graphs of any finite size by a mechanism of message passing (Gilmer et al., 2017). For every node to select, the authors feed to the network the graph representation of the problem - augmented with features indicating which of the nodes have already been visited and receive back an action value for every node. Action values are used to train the network through RL (Q-learning in particular) and the partial tour length is used as a reward.

This example does not do justice to the rich TSP literature that has developed far more advanced algorithms performing orders of magnitude better than ML ones. Nevertheless, the point we are trying to highlight here is that given a fixed context, and a decision to be made, ML can be used to discover new, potentially better performing policies. Even on stateof-the-art TSP algorithms (i.e., when exact solving is taken to its limits), many decisions are made in heuristic ways, e.g., cutting plane selection, thus leaving room for ML to assist in making these decisions.

Once again, we stress that learning a policy by experience is well described by the MDP framework of reinforcement learning, where an agent maximizes the return (defined in Section 2.2). By matching the reward signal with the optimization objective, the goal of the learning agent becomes to solve the problem, without assuming any expert knowledge. Some methods that were not presented as RL can also be cast in this MDP formulation, even if the optimization methods are not those of the RL community. For instance, part of the CO literature is dedicated to automatically build specialized heuristics for different problems. The heuristics are build by orchestrating a set of moves, or subroutines, from a pre-defined domain-specific collections. For instance, to tackle bipartite boolean quadratic programming problems, Karapetyan et al. (2017) represent this orchestration as a Markov chain where the states are the subroutines. One Markov chain is parametrized by its transition probabilities. Mascia et al. (2014), on the other hand, define valid succession of moves through a grammar, where words are moves and sentences correspond to heuristics. The authors introduce a parametric space to represent sentences of a grammar. In both cases, the setting is very close to the MDP of RL, but the parameters are learned 
though direct optimization of the performances of their associated heuristic through so-called automatic configuration tools (usually based on genetic or local search, and exploiting parallel computing). Note that the learning setting is rather simple as the parameters do not adapt to the problem instance, but are fixed for various clusters. From the ML point of view, this is equivalent to a piece-wise constant regression. If more complex models were to be used, direct optimization may not scale adequately to obtain good performances. The same approach to building heuristics can be brought one level up if, instead of orchestrating sets of moves, it arranges predefined heuristics. The resulting heuristic is then called a hyper-heuristic. Özcan et al. (2012) build a hyper-heuristic for examination timetabling by learning to combine existing heuristics. They use a bandit algorithm, a stateless form of RL (see Sutton and Barto, 2018, Chapter 2), to learn online a value function for each heuristic.

We close this section by noting that demonstration and experience are not mutually exclusive and most learning tasks can be tackled in both ways. In the case of selecting the branching variables in an MILP branch-andbound tree, one could adopt anyone of the two prior strategies. On the one hand, Marcos Alvarez et al. (2014, 2016, 2017); Khalil et al. (2016) estimate that strong branching is an effective branching strategy but computationally too expensive and build a machine learning model to approximate it. On the other hand, one could believe that no branching strategy is good enough and try to learn one from the ground up, for instance through reinforcement learning as suggested (but not implemented) in Khalil et al. (2016). An intermediary approach is proposed by Liberto et al. (2016). The authors recognize that, among traditional variable selection policies, the ones performing well at the top of the $\mathrm{B} \& \mathrm{~B}$ tree are not necessarily the same as the ones performing well deeper down. Hence, the authors learn a model to dynamically switch among predefined policies during $\mathrm{B} \& \mathrm{~B}$ based on the current state of the tree. While this seems like a case of imitation learning, given that traditional branching policies can be thought of as experts, this is actually not the case. In fact, the model is not learning from any expert, but really learning to choose between pre-existing policies. This is technically not a branching variable selection, but rather a branching heuristic selection policy. Each sub-tree is represented by a vector of handcrafted features, and a clustering of these vectors is performed. Similarly to what was detailed in the previous paragraph about the work of Karapetyan et al. (2017); Mascia et al. (2014), automatic configuration tools are then used to assign the best branching policy to each cluster. When branching at a given 
node, the cluster the closest to the current sub-tree is retrieved, and its assigned policy is used.

\subsection{Algorithmic structure}

In this section, we survey how the learned policies (whether from demonstration or experience) are combined with traditional $\mathrm{CO}$ algorithms, i.e., considering ML and explicit algorithms as building blocks, we survey how they can be laid out in different templates. The three following sections are not necessarily disjoint nor exhaustive but are a natural way to look at the literature.

\subsubsection{End to end learning}

A first idea to leverage machine learning to solve discrete optimization problems is to train the ML model to output solutions directly from the input instance, as shown in Figure 7.

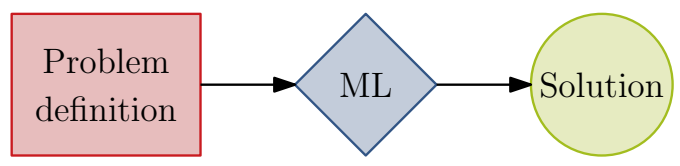

Figure 7: Machine learning acts alone to provide a solution to the problem.

This approach has been explored recently, especially on Euclidean TSPs. To tackle the problem with deep learning, Vinyals et al. (2015) introduce the pointer network wherein an encoder, namely an RNN, is used to parse all the TSP nodes in the input graph and produces an encoding (a vector of activations) for each of them. Afterward, a decoder, also an RNN, uses an attention mechanism similar to Bahdanau et al. (2015) (Section 2.2) over the previously encoded nodes in the graph to produce a probability distribution over these nodes (through the softmax layer previously illustrated in Figure 4). Repeating this decoding step, it is possible for the network to output a permutation over its inputs (the TSP nodes). This method makes it possible to use the network over different input graph sizes. The authors train the model through supervised learning with precomputed TSP solutions as targets. Bello et al. (2017) use a similar model and train it with reinforcement learning using the tour length as a reward signal. They address some limitations of supervised (imitation) learning, such as the need to compute optimal (or at least high quality) TSP solutions (the targets), that in turn, may be ill-defined when those solutions are not computed exactly, 
or when multiple solutions exist. Kool and Welling (2018) introduce more prior knowledge in the model using a GNN instead of an RNN to process the input. Emami and Ranka (2018) and Nowak et al. (2017) explore a different approach by directly approximating a double stochastic matrix in the output of the neural network to characterize the permutation. The work of Khalil et al. (2017a), introduced in Section 3.1.2, can also be understood as an end to end method to tackle the TSP, but we prefer to see it under the eye of Section 3.2.3. It is worth noting that tackling the TSP through ML is not new. Earlier work from the nineties focused on Hopfield neural networks and self organizing neural networks, the interested reader is referred to the survey of Smith (1999).

In another example, Larsen et al. (2018) train a neural network to predict the solution of a stochastic load planning problem for which a deterministic MILP formulation exists. Their main motivation is that the application needs to make decisions at a tactical level, i.e., under incomplete information, and machine learning is used to address the stochasticity of the problem arising from missing some of the state variables in the observed input. The authors use operational solutions, i.e., solutions to the deterministic version of the problem, and aggregate them to provide (tactical) solution targets to the ML model. As explained in their paper, the highest level of description of the solution is its cost, whereas the lowest (operational) is the knowledge of values for all its variables. Then, the authors place themselves in the middle and predict an aggregation of variables (tactical) that corresponds to the stochastic version of their specific problem. Furthermore, the nature of the application requires to output solutions in real time, which is not possible either for the stochastic version of the load planning problem or its deterministic variant when using state-of-the-art MILP solvers. Then, ML turns out to be suitable for obtaining accurate solutions with short computing times because some of the complexity is addressed offline, i.e., in the learning phase, and the run-time (inference) phase is extremely quick. Finally, note that in Larsen et al. (2018) an MLP, i.e., a feedforward neural network, is used to process the input instance as a vector, hence integrating very little prior knowledge about the problem structure.

\subsubsection{Learning to configure algorithms}

In many cases, using only machine learning to tackle the problem may not be the most suitable approach. Instead, ML can be applied to provide additional pieces of information to a $\mathrm{CO}$ algorithm as illustrated in Figure8, For example, ML can provide a parametrization of the algorithm (in a very 
broad sense).

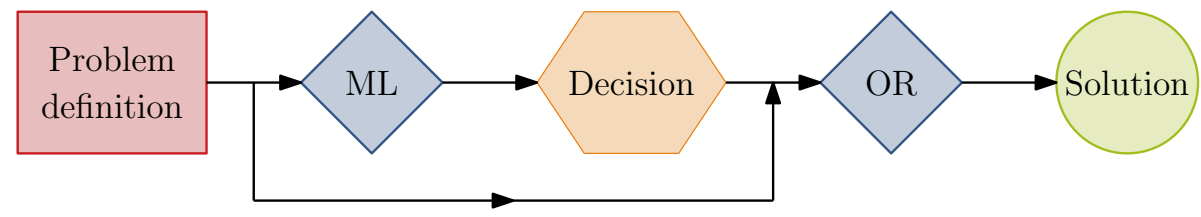

Figure 8: The machine learning model is used to augment an operation research algorithm with valuable pieces of information.

Algorithm configuration, detailed in Hoos (2012); Bischl et al. (2016), is a well studied area that captures the setting presented here. Complex optimization algorithms usually have a set of parameters left constant during optimization (in machine learning they are called hyper-parameters). For instance, this can be the aggressiveness of the pre-solving operations (usually controlled by a single parameter) of an MILP solver, or the learning rate / step size in gradient descent methods. Carefully selecting their value can dramatically change the performance of the optimization algorithm. Hence, the algorithm configuration community started looking for good default parameters. Then good default parameters for different cluster of similar problem instances. From the ML point of view, the former is a constant regression, while the second is a piece-wise constant nearest neighbors regression. The natural continuation was to learn a regression mapping problem instances to algorithm parameters.

In this context, Kruber et al. (2017) use machine learning on MILP instances to estimate beforehand whether or not applying a Dantzig-Wolf decomposition will be effective, i.e., will make the solving time faster. Decomposition methods can be powerful but deciding if and how to apply them depends on many ingredients of the instance and of its formulation and there is no clear cut way of optimally making such a decision. In their work, a data point is represented as a fixed length vector with features representing instance and tentative decomposition statistics. In another example, in the context of mixed-integer quadratic programming, Bonami et al. (2018) use machine learning to decide if linearizing the problem will solve faster. When the quadratic programming (QP) problem given by the relaxation is convex, i.e., the quadratic objective matrix is semidefinite positive, one could address the problem by a $\mathrm{B} \& \mathrm{~B}$ algorithm that solves $\mathrm{QP}$ relaxation 7 to provide lower bounds. Even in this convex case, it is not clear if QP B\&B

\footnotetext{
${ }^{7}$ Note that convex QPs can be solved in polynomial time.
} 
would solve faster than linearizing the problem (by using McCormick (1976) inequalities) and solving an equivalent MILP. This is why ML is a great candidate here to fill the knowledge gap. In both papers (Kruber et al., 2017; Bonami et al., 2018), the authors experiment with different ML models, such as support vector machines and random forests, as is good practice when no prior knowledge is embedded in the model.

The heuristic building framework used in Karapetyan et al. (2017) and Mascia et al. (2014), already presented in Section 3.1.2, can be understood under this eye. Indeed, it can be seen as a large parametric heuristic, configured by the transition probabilities in the former case, and by the parameter representing a sentence in the latter.

As previously stated, the parametrization of the $\mathrm{CO}$ algorithm provided by ML is to be understood in a very broad sense. For instance, in the case of radiation therapy for cancer treatment, Mahmood et al. (2018) use ML to produce candidate therapies that are afterward refined by a $\mathrm{CO}$ algorithm into a deliverable plan. Namely, a generative adversarial network (GAN) is used to color CT scan images into a potential radiation plan, then, inverse optimization (Ahuia and Orlin, 2001) is applied on the result to make the plan feasible (Chan et al., 2014). In general, GANs are made of two distinct networks: one (the generator) generates images, and another one (the discriminator) discriminates between the generated images and a dataset of real images. Both are trained alternatively: the discriminator through a usual supervised objective, while the generator is trained to fool the discriminator. In Mahmood et al. (2018), a particular type of GAN (conditional GAN) is used to provide coloring instead of random images. The interested reader is referred to Creswell et al. (2018) for an overview on GANs.

We end this section by noting that an ML model used for learning some representation may in turn use as features pieces of information given by another $\mathrm{CO}$ algorithm, such as the decomposition statistics used in Kruber et al. (2017), or the LP information in Bonami et al. (2018). Moreover, we remark that, in the satisfiability context, the learning of the type of algorithm to execute on a particular cluster of instances has been paired with the learning of the parameters of the algorithm itself, see, e.g., Ansótegui et al. (2017, 2019).

\subsubsection{Machine learning alongside optimization algorithms}

To generalize the context of the previous section to its full potential, one can build $\mathrm{CO}$ algorithms that repeatedly call an ML model throughout their 
execution, as illustrated in Figure 9. A master algorithm controls the highlevel structure while frequently calling an ML model to assist in lower level decisions. The key difference between this approach and the examples discussed in the previous section is that the same $M L$ model is used by the CO algorithm to make the same type of decisions a number of times in the order of the number of iterations of the algorithm. As in the previous section, nothing prevents one from applying additional steps before or after such an algorithm.

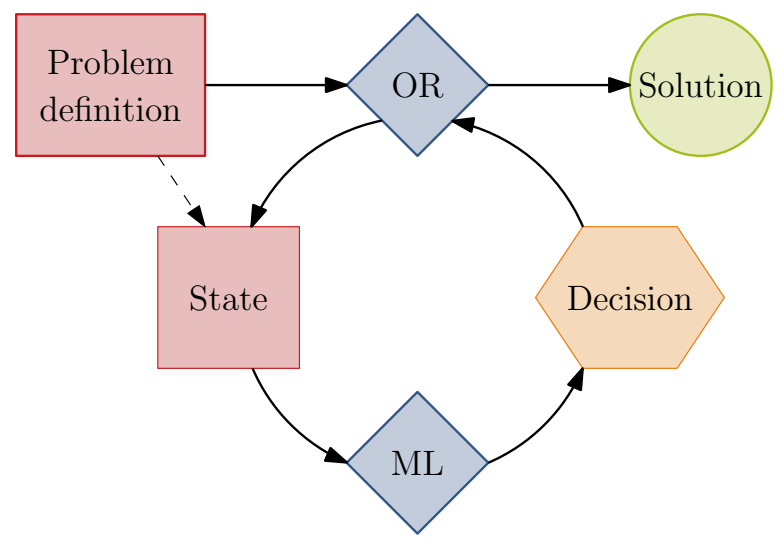

Figure 9: The combinatorial optimization algorithm repeatedly queries the same ML model to make decisions. The ML model takes as input the current state of the algorithm, which may include the problem definition.

This is clearly the context of the branch-and-bound tree for MILP, where we already mentioned how the task of selecting the branching variable is either too heuristic or too slow, and is therefore a good candidate for learning (Lodi and Zarpellon, 2017). In this case, the general algorithm remains a branch-and-bound framework, with the same software architecture and the same guarantees on lower and upper bounds, but the branching decisions made at every node are left to be learned. Likewise, the work of Hottung et al. (2017) learning both a branching policy and value network for heuristic tree search undeniably fits in this context. Another important aspect in solving MILPs is the use of primal heuristics, i.e., algorithms that are applied in the B\&B nodes to find feasible solutions, without guarantee of success. On top of their obvious advantages, good solutions also give tighter upper bounds (for minimization problems) on the solution value and make more pruning of the tree possible. Heuristics depend on the branching node (as branching fix some variables to specific values), so they need 
to be run frequently. However, running them too often can slow down the exploration of the tree, especially if their outcome is negative, i.e., no better upper bound is detected. Khalil et al. (2017b) build an ML model to predict whether or not running a given heuristic will yield a better solution than the best one found so far and then greedily run that heuristic whenever the outcome of the model is positive.

The approximation used by Baltean-Lugojan et al. (2018), already discussed in Section 3.2.1, is an example of predicting a high-level description of the solution to an optimization problem, namely the objective value. Nonetheless, the goal is to solve the original QP. Thus, the learned model is queried repeatedly to select promising cutting planes. The ML model is used only to select promising cuts, but once selected, cuts are added to the LP relaxation, thus embedding the ML outcome into an exact algorithm. This approach highlights promising directions for this type of algorithm. The decision learned is critical because adding the best cutting planes is necessary for solving the problem fast (or fast enough, because in the presence of NPhard problems, optimization may time out before any meaningful solving). At the same time, the approximate decision (often in the form of a probability) does not compromise the exactness of the algorithm: any cut added is guaranteed to be valid. This setting leaves room for ML to thrive, while reducing the need for guarantees from the ML algorithms (an active and difficult area of research). In addition, note that, the approach in Larsen et al. (2018) is part of a master algorithm in which the ML is iteratively invoked to make booking decisions in real time. The work of Khalil et al. (2017a), presented in Section 3.1.2, also belongs to this setting, even if the resulting algorithm is heuristic. Indeed, an ML model is asked to select the most relevant node, while a master algorithm maintains the partial tour, computes its length, etc. Because the master algorithm is very simple, it is possible to see the contribution as an end-to-end method, as stated in Section 3.2.1, but it can also be interpreted more generally as done here.

Presented in Section 3.1.2, and mentioned in the previous section, the Markov Chain framework for building heuristics from Karapetyan et al. (2017) can also be framed as repeated decisions. The transition matrix can be queried and sampled from in order to transition from one state to another, i.e., to make the low level decisions of choosing the next move. The three distinctions made in this Section 3.2 are general enough that they can overlap. Here, the fact that the model operates on internal state transitions, yet is learned globally, is what makes it hard to analyze.

Before ending this section, it is worth mentioning that learning recurrent algorithmic decisions is also used in the deep learning community, for 
instance in the field of meta-learning to decide how to apply gradient updates in stochastic gradient descent (Andrychowicz et al., 2016; Li and Malik, 2017; Wichrowska et al., 2017).

\section{Learning objective}

In the previous section, we have surveyed the existing literature by orthogonally grouping the main contributions of ML for CO into families of approaches, sometimes with overlaps. In this section, we formulate and study the objective that drives the learning process.

\subsection{Multi-instance formulation}

In the following, we introduce an abstract learning formulation (inspired from Bischl et al. (2016)). How would an ML practitioner compare optimization algorithms? Let us define $\mathcal{I}$ to be a set of problem instances, and $P$ a probability distribution over $\mathcal{I}$. These are the problems that we care about, weighted by a probability distribution, reflecting the fact that, in a practical application, not all problems are as likely. In practice, $\mathcal{I}$ or $P$ are inaccessible, but we can observe some samples from $P$, as motivated in the introduction with the Montreal delivery company. For a set of algorithms $\mathcal{A}$, let $m: \mathcal{I} \times \mathcal{A} \rightarrow \mathbb{R}$ be a measure of the performance of an algorithm on a problem instance (lower is better). This could be the objective value of the best solution found, but could also incorporate elements from optimality bounds, absence of results, running times, and resource usage. To compare $a_{1}, a_{2} \in \mathcal{A}$, an ML practitioner would compare $\mathbb{E}_{i \sim P} m\left(i, a_{1}\right)$ and $\mathbb{E}_{i \sim P} m\left(i, a_{2}\right)$, or equivalently

$$
\min _{a \in\left\{a_{1}, a_{2}\right\}} \mathbb{E}_{i \sim P} m(i, a) .
$$

Because measuring these quantities is not tractable, one will typically use empirical estimates instead, by using a finite dataset $D_{\text {train }}$ of independent instances sampled from $P$

$$
\min _{a \in\left\{a_{1}, a_{2}\right\}} \sum_{i \in D_{\text {train }}} \frac{1}{\left|D_{\text {train }}\right|} m(i, a) .
$$

This is intuitive and done in practice: collect a dataset of problem instances and compare say, average running times. Of course, such expectation can be computed for different datasets (different $\mathcal{I}$ 's and $P$ 's), and different measures (different $m$ 's). 
This is already a learning problem. The more general one that we want to solve through leaning is

$$
\min _{a \in \mathcal{A}} \mathbb{E}_{i \sim P} m(i, a) .
$$

Instead of comparing between two algorithms, we may compare among an uncountable, maybe non-parametric, space of algorithms. To see how we come up with so many algorithms, we have to look at the algorithms in Section 3, and think of the ML model space over which we learn as defining parametrizing the algorithm space $\mathcal{A}$. For instance, consider the case of learning a branching policy $\pi$ for B\&B. If we define the policy to be a neural network with a set of weights $\theta \in \mathbb{R}^{p}$, then we obtain a parametric $\mathrm{B} \& \mathrm{~B}$ algorithm $a\left(\pi_{\theta}\right)$ and (6) becomes

$$
\min _{\theta \in \mathbb{R}^{p}} \mathbb{E}_{i \sim P} m\left(i, a\left(\pi_{\theta}\right)\right) .
$$

Unfortunately, solving this problem is hard. On the one hand, the performance measure $m$ is most often not differentiable and without closed form expression. We discuss this in Section 4.2. On the other hand, computing the expectation in (6) is intractable. As in (5), one can use an empirical distribution using a finite dataset, but that leads to generalization considerations, as explained in Section 4.3 .

Before we move on, let us introduce a new element to make (6) more general. That formula suggests that, once given an instance, the outcome of the performance measure is deterministic. That is unrealistic for multiple reasons. The performance measure could itself incorporate some source of randomness due to external factors, for instance with running times which are hardware and system dependent. The algorithm could also incorporate non negligible sources of randomness, if it is designed to be stochastic, or if some operations are non deterministic, or to express the fact that the algorithm should be robust to the choice of some external parameters. Let $\tau$ be that source of randomness, $\pi \in \Pi$ the internal policy being learned, and $a(\pi, \tau)$ the resulting algorithm, then we can reformulate (6) as

$$
\min _{\pi \in \Pi} \mathbb{E}_{i \sim P}\left[\mathbb{E}_{\tau}[m(i, a(\pi, \tau)) \mid i]\right]
$$

In particular, when learning repeated decisions, as in Section 3.2.3, this source of randomness can be expressed along the trajectory followed in the MDP, using the dynamics of the environment $p\left(s^{\prime}, r \mid a, s\right)$ (see Figure 2). The addition made in (8) will be useful for the discussion on generalization in Section 4.3 . 


\subsection{Surrogate objectives}

In the previous section, we have formulated a proper learning objective. Here, we try to relate that objective to the learning methods of Section 3.1, namely, demonstration and experience. If the usual learning metrics of an ML model, e.g., accuracy for classification in supervised (imitation) learning, is improving, does it mean that the performance metric of (므) is also improving?

A straightforward approach for solving (8) is that of reinforcement learning (including direct optimization methods), as surveyed in Section 3.1.2. The objective from (6) can be optimized directly on experience data by matching the total return to the performance measure. Sometimes, a single final reward can naturally be decoupled across the trajectory. For instance, if the performance objective of a $\mathrm{B} \& \mathrm{~B}$ variable selection policy is to minimize the number of opened nodes, then the policy can receive a reward discouraging an increase in the number of nodes, hence giving an incentive to select variables that lead to pruning. However, that may not be always possible, leaving only the option of delaying a single reward to the end of the trajectory. This sparse reward setting is challenging for RL algorithms, and one might want to design a surrogate reward signal to encourage intermediate accomplishments. This introduces some discrepancies, and the policy being optimized may learn a behavior not intended by the algorithm designer. There is a priori no relationship between two reward signals. One needs to make use of their intuition to design surrogate signals, e.g., minimizing the number of $\mathrm{B} \& \mathrm{~B}$ nodes should lead to smaller running times. Reward shaping is an active area of research in RL, yet it is often performed by a number of engineering tricks.

In the case of learning a policy from a supervised signal from expert demonstration, the performance measure $m$ does not even appear in the learning problem that is solved. In this context, the goal is to optimize a policy $\pi \in \Pi$ in the action space to mimic an expert policy $\pi_{e}$ (as first introduced with Figure 5)

$$
\min _{\pi \in \Pi} \mathbb{E}_{i \sim P}\left[\mathbb{E}_{s}\left[\ell\left(\pi(s), \pi_{e}(s)\right) \mid i, \pi_{e}\right]\right],
$$

where $\ell$ is a task dependent loss (classification, regression, etc.). We have emphasized that the state $S$ is conditional, not only on the instance, but also on the expert policy $\pi_{e}$ used to collect the data. Intuitively, the better the ML model learns, i.e., the better the policy imitates the expert, the closer the final performance of the learned policy should be to the performance of the expert. Under some conditions, it is possible to relate the performance 
of the learned policy to the performance of the expert policy, but covering this aspect is out of the scope of this paper. The opposite is not true, if learning fails, the policy may still turn out to perform well (by encountering an alternative good decision). Indeed, when making a decision with high surrogate objective error, the learning will be fully penalized when, in fact, the decision could have good performances by the original metric. For that reason, it is capital to report the performance metrics. For example, we surveyed in Section 3.2.2 the work of Bonami et al. (2018) where the authors train a classifier to predict if a mixed integer quadratic problem instance should be linearized or not. The targets used for the learner are computed optimally by solving the problem instance in both configurations. Simply reporting the classification accuracy is not enough. Indeed, this metric gives no information on the impact a misclassification has on running times, the metric used to compute the targets. In the binary classification, a properly classified example could also happen to have unsignificant difference between the running times of the two configurations. To alleviate this issue, the authors also introduce a category where running times are not significatively different (and report the real running times). A continuous extension would be to learn a regression of the solving time. However, learning this regression now means that the final algorithm needs to optimize over the set of decisions to find the best one. In RL, this is analoguous to learning a value function (see Section 2.2). Applying the same reasoning to repeated decisions is better understood with the complete RL theory.

\subsection{On generalization}

In Section 4.1, we have claimed that the probability distribution in (므) is inaccessible and needs to be replaced by the empirical probability distribution over a finite dataset $D_{\text {train }}$. The optimization problem solved is

$$
\min _{a \in \mathcal{A}} \sum_{i \in D_{\text {train }}} \frac{1}{\left|D_{\text {train }}\right|} m(i, a) .
$$

As pointed out in Section 2.2, when optimizing over the empirical probability distribution, we risk having a low performance measure on the finite number of problem instances, regardless of the true probability distribution. In this case, the generalization error is high because of the discrepancy between the training performances and the true expected performances (overfitting). To control this aspect, a validation set $D_{\text {valid }}$ is introduced to compare a finite number of candidate algorithms based on estimates of generalization 
performances, and a test set $D_{\text {test }}$ is used for estimating the generalization performances of the selected algorithm.

In the following, we look more intuitively at generalization in ML for $\mathrm{CO}$, and its consequences. To make it easier, let us recall different learning scenarios. In the introduction, we have motivated the Montreal delivery company example, where the problems of interest are from an unknown probability distribution of Montreal TSPs. This is a very restricted set of problems, but enough to deliver value for this business. Much more ambitious, we may want our policy learned on a finite set of instances to perform well (generalize) to any "real-world" MILP instance. This is of interest if you are in the business of selling MILP solvers and want the branching policy to perform well for as many of your clients as possible. In both cases, generalization applies to the instances that are not known to the algorithm implementer. These are the only instances that we care about; the one used for training are already solved. The topic of probability distribution of instances also appears naturally in stochastic programming/optimization, where uncertainty about the problem is modeled through probability distributions. Scenario generation, an essential way to solve this type of optimization programs, require sampling from this distribution and solving the associated problem multiple times. Nair et al. (2018) take advantage of this repetitive process to learn an end-to-end model to solve the problem. Their model is composed of a local search and a local improvement policy and is trained through RL. Here, generalization means that, during scenario generation, the learned search beats other approaches, hence delivering an overall faster stochastic programming algorithm. In short, learning without generalization is pointless!

When the policy generalizes to other problem instances, it is no longer a problem if training requires additional computation for solving problem instances because, learning can be decoupled from solving as it can be done offline. This setting is promising as it could give a policy to use out of the box for similar instances, while keeping the learning problem to be handled beforehand while remaining hopefully reasonable. When the model learned is a simple mapping, as is the case in Section 3.2.2, generalization to new instances, as previously explained, can be easily understood. However, when learning sequential decisions, as in Section 3.2.3, there are intricate levels of generalization. We said that we want the policy to generalize to new instances, but the policy also needs to generalize to internal states of the algorithm for a single instance, even if the model can be learned from complete optimization trajectories, as formulated by (8). Indeed, complex algorithms can have unexpected sources of randomness, even if they are de- 
signed to be deterministic. For instance, a numerical approximation may perform differently if the version of some underlying numerical library is changed or because of asynchronous computing, such as when using Graphical Processing Units (Nagarajan et al., 2019). Furthermore, even if we can achieve perfect replicability, we do not want the branching policy to break if some other parameters of the solver are set (slightly) differently. At the very least, we want the policy to be robust to the choice of the random seed present in many algorithms, including MILP solvers. These parameters can therefore be modeled as random variables. Because of these nested levels of generalization, one appealing way to think about the training data from multiple instances is like separate tasks of a multi-task learning setting. The different tasks have underlying aspects in common, and they may also have their own peculiar quirks. One way to learn a single policy that generalizes within a distribution of instances is to take advantage of these commonalities. Generalization in RL remains a challenging topic, probably because of the fuzzy distinction between a multi-task setting, and a large environment encompassing all of the tasks.

Choosing how ambitious one should be in defining the characteristics of the distribution is a hard question. For instance, if the Montreal company expands its business to other cities, should they be considered as separate distributions, and learn one branching policy per city, or only a single one? Maybe one per continent? Generalization to a larger variety of instances is challenging and requires more advanced and expensive learning algorithms. Learning an array of ML models for different distributions asociated with a same task means of course more models to train, maintain, and deploy. The same goes with traditional CO algorithms, an MILP solver on its own is not the best performing algorithm to solve TSPs, but it works across all MILP problems. It is too early to provide insights about how broad the considered distributions should be, given the limited literature in the field. For scholars generating synthetic distributions, two intuitive axes of investigation are "structure" and "size". A TSP and a scheduling problem seem to have fairly different structure, and one can think of two planar euclidean TSPs to be way more similar. Still, two of these TSPs can have dramatically different sizes (number of nodes). For instance, Gasse et al. (2019) assess their methodology independently on three distributions. Each training dataset has a specific problem structure (set covering, combinatorial auction, and capacitated facility location), and a fixed problem size. The problem instance generators used are state-of-art and representative of realworld instances. Nonetheless, when they evaluate their learned algorithm, the authors push the test distributions to larger sizes. The idea behind 
this is to gauge if the model learned is able to generalize to a larger, more practical, distribution, or only perform well on the restricted distribution of problems of the same size. The answer is largely affirmative.

\subsection{Single instance learning}

An edge case that we have not much discussed yet is the single instance learning framework. This might be the case for instance for planning the design of a single factory. The factory would only be built once, with very peculiar requirements, and the planners are not interested to relate this to other problems. In this case, one can make as many runs (episodes) and as many calls to a potential expert or simulator as one wants, but ultimately one only cares about solving this one instance. Learning a policy for a single instance should require a simpler ML model, which could thus require less training examples. Nonetheless, in the single instance case, one learns the policy from scratch at every new instance, actually incorporating learning (not learned models but really the learning process itself) into the end algorithm. This means starting the timer at the beginning of learning and competing with other solvers to get the solution the fastest (or get the best results within a time limit). This is an edge scenario that can only be employed in the setting of the Section 3.2.3, where ML is embedded inside a $\mathrm{CO}$ algorithm; otherwise there would be only one training example! There is therefore no notion of generalization to other problem instances, so (6) is not the learning problem being solved. Nonetheless, the model still needs to generalize to unseen states of the algorithm. Indeed, if the model was learned from all states of the algorithm that are needed to solve the problem, then the problem is already solved at training time and learning is therefore fruitless. This is the methodology followed by Khalil et al. (2016), introduced in Section 3.1.1, to learn an instance-specific branching policy. The policy is learned from strong-branching at the top of the B\&B tree, but needs to generalize to the state of the algorithm at the bottom of the tree, where it is used. However, as for all $\mathrm{CO}$ algorithms, a fair comparison to another algorithm can only be done on an independent dataset of instances, as in (41). This is because through human trials and errors, the data used when building the algorithm leaks into the design of the algorithm, even without explicit learning components. 


\subsection{Fine tuning and meta-learning}

A compromise between instance-specific learning and learning a generic policy is what we typically have in multi-task learning: some parameters are shared across tasks and some are specific to each task. A common way to do that (in the transfer learning scenario) is to start from a generic policy and then adapt it to the particular instance by a form of fine-tuning procedure: training proceeds in two stages, first training the generic policy across many instances from the same distribution, and then continuing training on the examples associated with a given instance on which we are hoping to get more specialized and accurate predictions.

Machine learning advances in the areas of meta-learning and transfer learning are particularly interesting to consider here. Meta-learning considers two levels of optimization: the inner loop trains the parameters of a model on the training set in a way that depends on meta-parameters, which are themselves optimized in an outer loop (i.e., obtaining a gradient for each completed inner-loop training or update). When the outer loop's objective function is performance on a validation set, we end up training a system so that it will generalize well. This can be a successful strategy for generalizing from very few examples if we have access to many such training tasks. It is related to transfer learning, where we want that what has been learned in one or many tasks helps improve generalization on another. These approaches can help rapidly adapt to a new problem, which would be useful in the context of solving many MILP instances, seen as many related tasks.

To stay with the branching example on MILPs, one may not want the policy to perform well out of the box on new instances (from the given distribution). Instead, one may want to learn a policy offline that can be adapted to a new instance in a few training steps, every time it is given one. Similar topics have been explored in the context of automatic configuration tools. Fitzgerald et al. (2014) study the automatic configuration in the lifelong learning context (a form of sequential transfer learning). The automatic configuration algorithm is augmented with a set of previous configurations that are prioritized on any new problem instance. A score reflecting past performances is kept along every configuration. It is designed to retain configurations that performed well in the past, while letting new ones a chance to be properly evaluated. The automatic configuration optimization algorithm used by Lindauer and Hutter (2018) requires training an empirical cost model mapping the Cartesian product of parameter configurations and problem instances to expected algorithmic performance. Such a model is usually learned for every cluster of problem instance that requires config- 
uring. Instead, when presented with a new cluster, the authors combine the previously learned cost models and the new one to build an ensemble model. As done by Fitzgerald et al. (2014), the authors also build a set of previous configurations to prioritize, using an empirical cost model to fill the missing data. This setting, which is more general than not performing any adaptation of the policy, has potential for better generalization. Once again, the scale on which this is applied can vary depending on ambition. One can transfer on very similar instances, or learn a policy that transfers to a vast range of instances.

Meta-learning algorithms were first introduced in the 1990s (Bengio et al., 1991; Schmidhuber, 1992; Thrun and Pratt, 1998) and have since then become particularly popular in ML, including, but not limited to, learning a gradient update rule (Hochreiter et al., 2001; Andrychowicz et al., 2016), few shot learning (Ravi and Larochelle, 2017), and multi-task RL (Finn et al., 2017).

\subsection{Other metrics}

Other metrics from the process of learning itself are also relevant, such as how fast the learning process is, the sample complexity (number of examples required to properly fit the model), etc. As opposed to the metrics suggested earlier in this section, these metrics provide us with information not about final performance, but about offline computation or the number of training examples required to obtain the desired policy. This information is, of course, useful to calibrate the effort in integrating ML into CO algorithms.

\section{$5 \quad$ Methodology}

In the previous section, we have detailed the theoretical learning framework of using ML in CO algorithms. Here, we provide some additional discussion broadening some previously made claims.

\subsection{Demonstration and experience}

In order to learn a policy, we have highlighted two methodologies: demonstration, where the expected behavior is shown by an expert or oracle (sometimes at a considerable computational cost), and experience, where the policy is learned through trial and error with a reward signal.

In the demonstration setting, the performance of the learned policy is bounded by the expert, which is a limitation when the expert is not op- 
timal. More precisely, without a reward signal, the imitation policy can only hope to marginally outperform the expert (for example because the learner can reduce the variance of the answers across similarly-performing experts). The better the learning, the closer the performance of the learner to the expert's. This means that imitation alone should be used only if it is significantly faster than the expert to compute the policy. Furthermore, the performance of the learned policy may not generalize well to unseen examples and small variations of the task and may be unstable due to accumulation of errors. This is because in (9), the data was collected according to the expert policy $\pi_{e}$, but when run over multiple repeated decisions, the distribution of states becomes that of the learned policy. Some downsides of supervised (imitation) learning can be overcome with more advanced algorithms, including active methods to query the expert as an oracle to improve behavior in uncertain states. The part of imitation learning presented here is limited compared to the current literature in ML.

On the contrary, with a reward, the algorithm learns to optimize for that signal and can potentially outperform any expert, at the cost of a much longer training time. Learning from a reward signal (experience) is also more flexible when multiple decisions are (almost) equally good in comparison with an expert that would favor one (arbitrary) decision. Experience is not without flaws. In the case where policies are approximated (e.g., with a neural network), the learning process may get stuck around poor solutions if exploration is not sufficient or solutions which do not generalize well are found. Furthermore, it may not always be straightforward to define a reward signal. For instance, sparse rewards may be augmented using reward shaping or a curriculum in order to value intermediate accomplishments (see Section 2.2).

Often, it is a good idea to start learning from demonstrations by an expert, then refine the policy using experience and a reward signal. This is what was done in the original AlphaGo paper (Silver et al., 2016), where human knowledge is combined with reinforcement learning. The reader is referred to Hussein et al. (2017) for a survey on imitation learning covering most of the discussion in this section.

\subsection{Partial observability}

We mentioned in section 2.2 that sometimes the states of an MDP are not fully observed and the Markov property does not hold, i.e., the probability of the next observation, conditioned on the current observation and action, is not equal to the probability of the next observation, conditioned on all 
past observations and actions. An immediate example of this can be found in any environment simulating physics: a single frame/image of such an environment is not sufficient to grasp notions such as velocity and is therefore not sufficient to properly estimate the future trajectory of objects. It turns out that, on real applications, partial observability is closer to the norm than to the exception, either because one does not have access to a true state of the environment, or because it is not computationally tractable to represent and needs to be approximated. A straightforward way to tackle the problem is to compress all previous observations using an RNN. This can be applied in the imitation learning setting, as well as in RL, for instance by learning a recurrent policy (Wierstra et al., 2010).

How does this apply in the case where we want to learn a policy function making decisions for a $\mathrm{CO}$ algorithm? On the one hand, one has full access to the state of the algorithm because it is represented in exact mathematical concepts, such as constraints, cuts, solutions, B\&B tree, etc. On the other hand, these states can be exponentially large. This is an issue in terms of computations and generalization. Indeed, if one does want to solve problems quickly, one needs to have a policy that is also fast to compute, especially if it is called frequently as is the case for, say, branching decisions. Furthermore, considering too high-dimensional states is also a statistical problem for learning, as it may dramatically increase the required number of samples, decrease the learning speed, or fail altogether. Hence, it is necessary to keep these aspects in mind while experimenting with different representations of the data.

\subsection{Exactness and approximation}

In the different examples we have surveyed, ML is used in both exact and heuristic frameworks, for example Baltean-Lugojan et al. (2018) and Larsen et al. (2018), respectively. Getting the output of an ML model to respect advanced types of constraints is a hard task. In order to build exact algorithms with ML components, it is necessary to apply the ML where all possible decisions are valid. Using only ML as surveyed in Section 3.2.1 cannot give any optimality guarantee, and only weak feasibility guarantees (see Section 6.1). However, applying ML to select or parametrize a $\mathrm{CO}$ algorithm as in Section 3.2 .2 will keep exactness if all possible choices that ML discriminate lead to complete algorithms. Finally, in the case of repeated interactions between ML and CO surveyed in Section 3.2.3, all possible decisions must be valid. For instance, in the case of MILPs, this includes branching among fractional variables of the LP relaxation, selecting 
the node to explore among open branching nodes (He et al., 2014), deciding on the frequency to run heuristics on the B\&B nodes (Khalil et al., 2017b), selecting cutting planes among valid inequalities (Baltean-Lugojan et al., 2018), removing previous cutting planes if they are not original constraints or branching decision, etc. A counter-example can be found in the work of Hottung et al. (2017), presented in Section 3.1.1. In their branch-an-bound framework, bounding is performed by an approximate ML model that can overestimate lower bounds, resulting in invalid pruning. The resulting algorithm is therefore not an exact one.

\section{Challenges}

In this section, we are reviewing some of the algorithmic concepts previously introduced by taking the viewpoint of their associated challenges.

\subsection{Feasibility}

In Section 3.2.1, we pointed out how ML can be used to directly output solutions to optimization problems. Rather than learning the solution, it would be more precise to say that the algorithm is learning a heuristic. As already repeatedly noted, the learned algorithm does not give any guarantee in terms of optimality, but it is even more critical that feasibility is not guaranteed either. Indeed, we do not know how far the output of the heuristic is from the optimal solution, or if it even respects the constraints of the problem. This can be the case for every heuristic and the issue can be mitigated by using the heuristic within an exact optimization algorithm (such as branch and bound).

Finding feasible solutions is not an easy problem (theoretically NP-hard for MILPs), but it is even more challenging in ML, especially by using neural networks. Indeed, trained with gradient descent, neural architectures must be designed carefully in order not to break differentiability. For instance, both pointer networks (Vinvals et al., 2015) and the Sinkhorn layer (Emami and Ranka, 2018) are complex architectures used to make a network output a permutation, a constraint easy to satisfy when writing a classical CO heuristic.

\subsection{Modelling}

In ML, in general, and in deep learning, in particular, we know some good prior for some given problems. For instance, we know that a CNN is an 
architecture that will learn and generalize more easily than others on image data. The problems studied in $\mathrm{CO}$ are different from the ones currently being addressed in ML, where most successful applications target natural signals. The architectures used to learn good policies in combinatorial optimization might be very different from what is currently used with deep learning. This might also be true in more subtle or unexpected ways: it is conceivable that, in turn, the optimization components of deep learning algorithms (say, modifications to SGD) could be different when deep learning is applied to the $\mathrm{CO}$ context.

Current deep learning already provides many techniques and architectures for tackling problems of interest in CO. As pointed out in section 2.2, techniques such as parameter sharing made it possible for neural networks to process sequences of variable length with RNNs or, more recently, to process graph structured data through GNNs. Processing graph data is of uttermost importance in CO because many problems are formulated (represented) on graphs. For a very general example, Selsam et al. (2018) represent a satisfiability problem using a bipartite graph on variables and clauses. This can generalize to MILPs, where the constraint matrix can be represented as the adjacency matrix of a bipartite graph on variables and constraints, as done in Gasse et al. (2019).

\subsection{Scaling}

Scaling to larger problems can be a challenge. If a model trained on instances up to some size, say TSPs up to size fifty nodes, is evaluated on larger instances, say TSPs of size a hundred, five hundred nodes, etc, the challenge exists in terms of generalization, as mentioned in Section 4.3. Indeed, all of the papers tackling TSP through ML and attempting to solve larger instances see degrading performance as size increases much beyond the sizes seen during training (Vinvals et al., 2015; Bello et al., 2017; Khalil et al., 2017a; Kool and Welling, 2018). To tackle this issue, one may try to learn on larger instances, but this may turn out to be a computational and generalization issue. Except for very simple ML models and strong assumptions about the data distribution, it is impossible to know the computational complexity and the sample complexity, i.e. the number of observations that learning requires, because one is unaware of the exact problem one is trying to solve, i.e., the true data generating distribution. 


\subsection{Data generation}

Collecting data (for example instances of optimization problems) is a subtle task. Larsen et al. (2018) claim that "sampling from historical data is appropriate when attempting to mimic a behavior reflected in such data". In other words, given an external process on which we observe instances of an optimization problem, we can collect data to train some policy needed for optimization, and expect the policy to generalize on future instances of this application. A practical example would be a business that frequently encounters optimization problems related to their activities, such as the Montreal delivery company example used in the introduction.

In other cases, i.e., when we are not targeting a specific application for which we would have historical data, how can we proactively train a policy for problems that we do not yet know of? As partially discussed in Section 4.3, we first need to define to which family of instances we want to generalize over. For instance, we might decide to learn a cutting plane selection policy for Euclidian TSP problems. Even so, it remains a complex effort to generate problems that capture the essence of real applications. Moreover, CO problems are high dimensional, highly structured, and troublesome to visualize. The sole exercise of generating graphs is already a complicated one! The topic has nonetheless received some interest. Smith-Miles and Bowly (2015) claim that the confidence we can put in an algorithm "depends on how carefully we select test instances", but note however that too often, a new algorithm is claimed "to be superior by showing that it outperforms previous approaches on a set of well-studied instances". The authors propose a problem instance generating method that consists of: defining an instance feature space, visualizing it in two dimensions (using dimensionality reduction techniques such as principal component analysis), and using an evolutionary algorithm to drive the instance generation toward a pre-defined sub-space. The authors argue that the method is successful if the easy and hard instances can be easily separated in the reduced instance space. The methodology is then fruitfully applied to graph-based problems, but would require redefining evolution primitives in order to be applied to other type of problems. On the contrary, Malitsky et al. (2016) propose a method to generate problem instances from the same probability distribution, in that case, the one of "industrial" boolean satisfiability problem instances. The authors use a large neighborhood search, using destruction and reparation primitives, to search for new instances. Some instance features are computed to classify whether the new instances fall under the same cluster as the target one. 
Deciding how to represent the data is also not an easy task, but can have a dramatic impact on learning. For instance, how does one properly represent a $\mathrm{B} \& \mathrm{~B}$ node, or even the whole $\mathrm{B} \& \mathrm{~B}$ tree? These representations need to be expressive enough for learning, but at the same time, concise enough to be used frequently without excessive computations.

\section{Conclusions}

We have surveyed and highlighted how machine learning can be used to build combinatorial optimization algorithms that are partially learned. We have suggested that imitation learning alone can be valuable if the policy learned is significantly faster to compute than the original one provided by an expert, in this case a combinatorial optimization algorithm. On the contrary, models trained with a reward signal have the potential to outperform current policies, given enough training and a supervised initialization. Training a policy that generalizes to unseen problems is a challenge, this is why we believe learning should occur on a distribution small enough that the policy could fully exploit the structure of the problem and give better results. We believe end-to-end machine learning approaches to combinatorial optimization can be improved by using machine learning in combination with current combinatorial optimization algorithms to benefit from the theoretical guarantees and state-of-the-art algorithms already available.

Other than performance incentives, there is also interest in using machine learning as a modelling tool for discrete optimization, as done by Lombardi and Milano (2018), or to extract intuition and knowledge about algorithms as mentioned in Bonami et al. (2018); Khalil et al. (2017a).

Although most of the approaches we discussed in this paper are still at an exploratory level of deployment, at least in terms of their use in generalpurpose (commercial) solvers, we strongly believe that this is just the beginning of a new era for combinatorial optimization algorithms.

\section{Acknowledgments}

The authors are grateful to Emma Frejinger, Simon Lacoste-Julien, Jason Jo, Laurent Charlin, Matteo Fischetti, Rémi Leblond, Michela Milano, Sébastien Lachapelle, Eric Larsen, Pierre Bonami, Martina Fischetti, Elias Khalil, Bistra Dilkina, Sebastian Pokutta, Marco Lübbecke, Andrea Tramontani, Dimitris Bertsimas and the entire CERC team for endless discussions on the subject and for reading and commenting a preliminary version 
of the paper.

\section{References}

Ahuja, R. K. and Orlin, J. B. (2001). Inverse Optimization. Operations Research, 49(5):771-783.

Andrychowicz, M., Denil, M., Gómez, S., Hoffman, M. W., Pfau, D., Schaul, T., Shillingford, B., and de Freitas, N. (2016). Learning to learn by gradient descent by gradient descent. In Lee, D. D., Sugiyama, M., Luxburg, U. V., Guyon, I., and Garnett, R., editors, Advances in Neural Information Processing Systems 29, pages 3981-3989. Curran Associates, Inc.

Ansótegui, C., Heymann, B., Pon, J., Sellmann, M., and Tierney, K. (2019). Hyper-Reactive Tabu Search for MaxSAT. In Battiti, R., Brunato, M., Kotsireas, I., and Pardalos, P. M., editors, Learning and Intelligent $O p$ timization, Lecture Notes in Computer Science, pages 309-325. Springer International Publishing.

Ansótegui, C., Pon, J., Sellmann, M., and Tierney, K. (2017). Reactive Dialectic Search Portfolios for MaxSAT. In Thirty-First AAAI Conference on Artificial Intelligence.

Applegate, D., Bixby, R., Chvátal, V., and Cook, W. (2007). The traveling salesman problem. A computational study. Princeton University Press.

Bahdanau, D., Cho, K., and Bengio, Y. (2015). Neural machine translation by jointly learning to align and translate. In ICLR'2015, arXiv:1409.0473.

Baltean-Lugojan, R., Misener, R., Bonami, P., and Tramontani, A. (2018). Strong sparse cut selection via trained neural nets for quadratic semidefinite outer-approximations. Technical report, Imperial College, London.

Bello, I., Pham, H., Le, Q. V., Norouzi, M., and Bengio, S. (2017). Neural Combinatorial Optimization with Reinforcement Learning. In International Conference on Learning Representations.

Bengio, Y., Bengio, S., Cloutier, J., and Gecsei, J. (1991). Learning a synaptic learning rule. In $I J C N N$, pages II-A969.

Bischl, B., Kerschke, P., Kotthoff, L., Lindauer, M., Malitsky, Y., Fréchette, A., Hoos, H., Hutter, F., Leyton-Brown, K., Tierney, K., and Vanschoren, 
J. (2016). ASlib: A benchmark library for algorithm selection. Artificial Intelligence, 237:41-58.

Bishop, C. M. (2006). Pattern Recognition and Machine Learning. springer.

Bonami, P., Lodi, A., and Zarpellon, G. (2018). Learning a Classification of Mixed-Integer Quadratic Programming Problems. In Integration of Constraint Programming, Artificial Intelligence, and Operations Research, Lecture Notes in Computer Science, pages 595-604. Springer, Cham.

Chan, T. C. Y., Craig, T., Lee, T., and Sharpe, M. B. (2014). Generalized Inverse Multiobjective Optimization with Application to Cancer Therapy. Operations Research, 62(3):680-695.

Conforti, M., Conrnuéjols, G., and Zambelli, G. (2014). Integer Programming. Springer.

Creswell, A., White, T., Dumoulin, V., Arulkumaran, K., Sengupta, B., and Bharath, A. A. (2018). Generative Adversarial Networks: An Overview. IEEE Signal Processing Magazine, 35(1):53-65.

Dai, H., Dai, B., and Song, L. (2016). Discriminative Embeddings of Latent Variable Models for Structured Data. In Balcan, M. F. and Weinberger, K. Q., editors, Proceedings of The 33rd International Conference on Machine Learning, volume 48 of Proceedings of Machine Learning Research, pages 2702-2711, New York, New York, USA. PMLR.

Dey, S. and Molinaro, M. (2018). Theoretical challenges towards cuttingplane selection. Mathematical Programming, 170:237-266.

Emami, P. and Ranka, S. (2018). Learning Permutations with Sinkhorn Policy Gradient. arXiv:1805.0\%010 [cs, stat].

Finn, C., Abbeel, P., and Levine, S. (2017). Model-Agnostic Meta-Learning for Fast Adaptation of Deep Networks. In Precup, D. and Teh, Y. W., editors, Proceedings of the 34th International Conference on Machine Learning, volume 70 of Proceedings of Machine Learning Research, pages 11261135, International Convention Centre, Sydney, Australia. PMLR.

Fischetti, M. and Lodi, A. (2011). Heuristics in Mixed Integer Programming, volume 3, pages 2199-2204. Wiley Online Library.

Fitzgerald, T., Malitsky, Y., O'Sullivan, B., and Tierney, K. (2014). ReACT: Real-Time Algorithm Configuration through Tournaments. In Seventh Annual Symposium on Combinatorial Search. 
Fortun, M. and Schweber, S. S. (1993). Scientists and the legacy of world war ii: The case of operations research (or). Social Studies of Science, 23(4):595-642.

Gasse, M., Chételat, D., Ferroni, N., Charlin, L., and Lodi, A. (2019). Exact combinatorial optimization with graph convolutional neural networks. arXiv preprint arXiv:1906.01629.

Gendreau, M. and Potvin, J.-Y., editors (2010). Handbook of metaheuristics, volume 2. Springer.

Gilmer, J., Schoenholz, S. S., Riley, P. F., Vinyals, O., and Dahl, G. E. (2017). Neural Message Passing for Quantum Chemistry. In Precup, D. and Teh, Y. W., editors, Proceedings of the 34th International Conference on Machine Learning, volume 70 of Proceedings of Machine Learning Research, pages 1263-1272, International Convention Centre, Sydney, Australia. PMLR.

Goodfellow, I., Bengio, Y., and Courville, A. (2016). Deep Learning. MIT press.

He, H., Daume III, H., and Eisner, J. M. (2014). Learning to Search in Branch and Bound Algorithms. In Ghahramani, Z., Welling, M., Cortes, C., Lawrence, N. D., and Weinberger, K. Q., editors, Advances in Neural Information Processing Systems 27, pages 3293-3301. Curran Associates, Inc.

Hochreiter, S., Younger, A. S., and Conwell, P. R. (2001). Learning to learn using gradient descent. In Dorffner, G., Bischof, H., and Hornik, K., editors, Artificial Neural Networks - ICANN 2001, pages 87-94, Berlin, Heidelberg. Springer Berlin Heidelberg.

Hoos, H. H. (2012). Automated Algorithm Configuration and Parameter Tuning. In Hamadi, Y., Monfroy, E., and Saubion, F., editors, Autonomous Search, pages 37-71. Springer Berlin Heidelberg, Berlin, Heidelberg.

Hottung, A., Tanaka, S., and Tierney, K. (2017). Deep Learning Assisted Heuristic Tree Search for the Container Pre-marshalling Problem. arXiv:1709.09972 [cs]. arXiv: 1709.09972.

Hussein, A., Gaber, M. M., Elyan, E., and Jayne, C. (2017). Imitation Learning: A Survey of Learning Methods. ACM Computing Surveys, $50(2): 21: 1-21: 35$. 
Karapetyan, D., Punnen, A. P., and Parkes, A. J. (2017). Markov Chain methods for the Bipartite Boolean Quadratic Programming Problem. European Journal of Operational Research, 260(2):494-506.

Khalil, E., Dai, H., Zhang, Y., Dilkina, B., and Song, L. (2017a). Learning Combinatorial Optimization Algorithms over Graphs. In Guyon, I., Luxburg, U. V., Bengio, S., Wallach, H., Fergus, R., Vishwanathan, S., and Garnett, R., editors, Advances in Neural Information Processing Systems 30, pages 6348-6358. Curran Associates, Inc.

Khalil, E. B., Bodic, P. L., Song, L., Nemhauser, G., and Dilkina, B. (2016). Learning to Branch in Mixed Integer Programming. In Proceedings of the Thirtieth AAAI Conference on Artificial Intelligence, AAAI'16, pages 724-731, Phoenix, Arizona. AAAI Press.

Khalil, E. B., Dilkina, B., Nemhauser, G. L., Ahmed, S., and Shao, Y. (2017b). Learning to Run Heuristics in Tree Search. In Proceedings of the Twenty-Sixth International Joint Conference on Artificial Intelligence, IJCAI-17, pages 659-666.

Kool, W. W. M. and Welling, M. (2018). Attention Solves Your TSP, Approximately. arXiv:1803.08475 [cs, stat].

Kruber, M., Lübbecke, M. E., and Parmentier, A. (2017). Learning When to Use a Decomposition. In Integration of AI and OR Techniques in Constraint Programming, Lecture Notes in Computer Science, pages 202210. Springer, Cham.

Larsen, E., Lachapelle, S., Bengio, Y., Frejinger, E., Lacoste-Julien, S., and Lodi, A. (2018). Predicting Solution Summaries to Integer Linear Programs under Imperfect Information with Machine Learning. arXiv:1807.11876 [cs, stat].

Larson, R. C. and Odoni, A. R. (1981). Urban operations research. Number Monograph.

Li, K. and Malik, J. (2017). Learning to Optimize Neural Nets. arXiv:1703.00441 [cs, math, stat].

Liberto, G. D., Kadioglu, S., Leo, K., and Malitsky, Y. (2016). DASH: Dynamic Approach for Switching Heuristics. European Journal of Operational Research, 248(3):943-953. 
Lindauer, M. and Hutter, F. (2018). Warmstarting of Model-Based Algorithm Configuration. In Thirty-Second AAAI Conference on Artificial Intelligence.

Lodi, A. (2009). MIP computation. In Jünger, M., Liebling, T., Naddef, D., Nemhauser, G., Pulleyblank, W., Reinelt, G., Rinaldi, G., and Wolsey, L., editors, 50 Years of Integer Programming 1958-2008, pages 619-645. Springer-Verlag.

Lodi, A. and Zarpellon, G. (2017). On learning and branching: A survey. TOP, 25(2):207-236.

Lombardi, M. and Milano, M. (2018). Boosting Combinatorial Problem Modeling with Machine Learning. In Proceedings of the Twenty-Seventh International Joint Conference on Artificial Intelligence, IJCAI-18, pages 5472-5478. International Joint Conferences on Artificial Intelligence Organization.

Mahmood, R., Babier, A., McNiven, A., Diamant, A., and Chan, T. C. Y. (2018). Automated Treatment Planning in Radiation Therapy using Generative Adversarial Networks. In Proceedings of Machine Learning for Health Care, volume 85 of Proceedings of Machine Learning Research.

Malitsky, Y., Merschformann, M., O’Sullivan, B., and Tierney, K. (2016). Structure-Preserving Instance Generation. In Festa, P., Sellmann, M., and Vanschoren, J., editors, Learning and Intelligent Optimization, Lecture Notes in Computer Science, pages 123-140. Springer International Publishing.

Marcos Alvarez, A., Louveaux, Q., and Wehenkel, L. (2014). A supervised machine learning approach to variable branching in branch-and-bound. Technical report, Université de Liège.

Marcos Alvarez, A., Louveaux, Q., and Wehenkel, L. (2017). A Machine Learning-Based Approximation of Strong Branching. INFORMS Journal on Computing, 29(1):185-195.

Marcos Alvarez, A., Wehenkel, L., and Louveaux, Q. (2016). Online Learning for Strong Branching Approximation in Branch-and-Bound. Technical report, Université de Liège.

Mascia, F., López-Ibáñez, M., Dubois-Lacoste, J., and Stützle, T. (2014). Grammar-based generation of stochastic local search heuristics through 
automatic algorithm configuration tools. Computers \& Operations Research, 51:190-199.

McCormick, G. P. (1976). Computability of global solutions to factorable nonconvex programs: Part I - Convex underestimating problems. Mathematical Programming, 10(1):147-175.

Murphy, K. P. (2012). Machine Learning: A Probabilistic Perspective. MIT press.

Nagarajan, P., Warnell, G., and Stone, P. (2019). Deterministic implementations for reproducibility in deep reinforcement learning. In AAAI 2019 Workshop on Reproducible AI.

Nair, V., Dvijotham, D., Dunning, I., and Vinyals, O. (2018). Learning fast optimizers for contextual stochastic integer programs. In Conference on Uncertainty in Artifical Intelligence, pages 591-600.

Nowak, A., Villar, S., Bandeira, A. S., and Bruna, J. (2017). A Note on Learning Algorithms for Quadratic Assignment with Graph Neural Networks. arXiv:1706.07450 [cs, stat].

Ravi, S. and Larochelle, H. (2017). Optimization as a model for few-shot learning. In International Conference on Learning Representations.

Schmidhuber, J. (1992). Learning to control fast-weight memories: An alternative to dynamic recurrent networks. Neural Computation, 4(1):131-139.

Selsam, D., Lamm, M., Bünz, B., Liang, P., de Moura, L., and Dill, D. L. (2018). Learning a SAT Solver from Single-Bit Supervision. arXiv:1802.03685 [cs].

Silver, D., Huang, A., Maddison, C. J., Guez, A., Sifre, L., van den Driessche, G., Schrittwieser, J., Antonoglou, I., Panneershelvam, V., Lanctot, M., Dieleman, S., Grewe, D., Nham, J., Kalchbrenner, N., Sutskever, I., Lillicrap, T., Leach, M., Kavukcuoglu, K., Graepel, T., and Hassabis, D. (2016). Mastering the game of Go with deep neural networks and tree search. Nature, 529(7587):484-489.

Smith, K. A. (1999). Neural Networks for Combinatorial Optimization: A Review of More Than a Decade of Research. INFORMS Journal on Computing, 11(1):15-34. 
Smith-Miles, K. and Bowly, S. (2015). Generating new test instances by evolving in instance space. Computers $\&$ Operations Research, 63:102113.

Sutton, R. S. and Barto, A. G. (2018). Reinforcement Learning: An Introduction. MIT press Cambridge, second edition.

Thrun, S. and Pratt, L. Y., editors (1998). Learning to Learn. Kluwer Academic.

Vaswani, A., Shazeer, N., Parmar, N., Uszkoreit, J., Jones, L., Gomez, A. N., Kaiser, L., and Polosukhin, I. (2017). Attention is All you Need. In Guyon, I., Luxburg, U. V., Bengio, S., Wallach, H., Fergus, R., Vishwanathan, S., and Garnett, R., editors, Advances in Neural Information Processing Systems 30, pages 5998-6008. Curran Associates, Inc.

Veličković, P., Cucurull, G., Casanova, A., Romero, A., Liò, P., and Bengio, Y. (2018). Graph attention networks. In International Conference on Learning Representations.

Vinyals, O., Fortunato, M., and Jaitly, N. (2015). Pointer Networks. In Cortes, C., Lawrence, N. D., Lee, D. D., Sugiyama, M., and Garnett, R., editors, Advances in Neural Information Processing Systems 28, pages 2692-2700. Curran Associates, Inc.

Wichrowska, O., Maheswaranathan, N., Hoffman, M. W., Colmenarejo, S. G., Denil, M., de Freitas, N., and Sohl-Dickstein, J. (2017). Learned Optimizers that Scale and Generalize. In Precup, D. and Teh, Y. W., editors, Proceedings of the 34 th International Conference on Machine Learning, volume 70 of Proceedings of Machine Learning Research, pages 37513760, International Convention Centre, Sydney, Australia. PMLR.

Wierstra, D., Förster, A., Peters, J., and Schmidhuber, J. (2010). Recurrent policy gradients. Logic Journal of the IGPL, 18(5):620-634.

Wolsey, L. A. (1998). Integer Programming. Wiley.

Özcan, E., Misir, M., Ochoa, G., and Burke, E. K. (2012). A Reinforcement Learning: Great-Deluge Hyper-Heuristic for Examination Timetabling. Modeling, Analysis, and Applications in Metaheuristic Computing: Advancements and Trends, pages 34-55. 